\title{
A review on nanocellulosic fibres as new material for sustainable packaging: process and applications
}

\begin{abstract}
The demand for exploring advanced and eco-friendly sustainable packaging materials with superior physical, mechanical and barrier properties is increasing. The materials that are currently used in packaging for food, beverage, medical and pharmaceutical products, as well as in industrial applications, are non-degradable, and thus, these materials are raising environmental pollution concerns. Numerous studies have been conducted on the utilization of bio-based materials in the pursuit of developing sustainable packaging materials. Although significant improvements have been achieved, a balance among environmental concerns, economic considerations and product packaging performance is still lacking. This is likely due to bio-based materials being used in product packaging applications without a proper design. The present review article intends to summarize the information regarding the potential applications of cellulosic nanofiber for the packaging. The importance of the design process, its principles and the challenges of design process for sustainable packaging are also summarized in this review. Overall it can be concluded that scientists, designers and engineers all are necessarily required to contribute towards research in order to commercially exploit cellulose nanofiber for sustainable packaging.
\end{abstract}

Keyword: Cellulose nanofiber; Design process; Eco-friendly packaging; Natural fiber; Sustainable material 
